teraction, satisfies time-reversal invariance to within the accuracy of the measurements.

It is a pleasure to recognize the encouragement and support of A. M. Weinberg, A. H. Snell, and R. S. Livingston. We wish to thank H. G. MacPherson and $\mathrm{H}$. Beutler for providing us with ${ }^{13} \mathrm{C}$ target. The assistance of $\mathrm{R}$. O. Ginaven, E. V. Hungerford, III, B. J. Morton, and R. W. Rutkowski during the runs is gratefully acknowledged. We also thank the Oak Ridge Isochronous Cyclotron staff, M. B. Marshall, and A. W. Alexander for their essential contributions.

*Research sponsored by the U. S. Atomic Energy Commission under contract with Union Carbide Corporation.

${ }^{1}$ J. H. Christenson, J. W. Cronin, V. L. Fitch, and R. Turlay, Phys. Rev. Letters 13, 138 (1964).

${ }^{2}$ W. G. Weitkamp, D. W. Storm, D. C. Shreve, W. J. Braithwaite, and D. Bodansky, Phys. Rev. 165, 1233 (1968); W. von Witsch, A. Richter, and P. von Bren- tano, Phys. Rev. 169, 923 (1967); S. T. Thornton, C. M. Jones, J. K. Bair, M. D. Mancusi, and H. B. Willard, Phys. Rev. Letters 21, 447 (1968).

${ }^{3}$ R. Handler, S. C. Wright, L. Pondrom, P. Limon, S. Olsen, and P. Kloeppel, Phys. Rev. Letters 19, 933 (1967).

${ }^{4}$ E. J. Squires, Progr. Nucl. Phys. 8,47 (1960).

${ }^{5}$ V. M. Lobashov, V. Z. Nazarenko, L. F. Saenko, L. M. Smotritsky, and G. I. Kharkevitch, Zh. Eksperim. i Teor. Fiz.-Pis'ma Redakt. 5, 73 (1967) [translation: JETP Letters $\underline{5}, 59$ (1967)].

${ }^{6}$ P. Hillman, A. Johansson, and G. Tibell, Phys. Rev. 110, 1218 (1958); A. Abashian and E. M. Hafner, Phys. Rev. Letters 1, 255 (1958); L. Rosen and J. E. Brolley, Jr., Phys. Rev. Letters 2 , 98 (1959); C. F. Hwang, T. R. Ophel, E. H. Thorndike, and R. Wilson, Phys. Rev. 119, 352 (1960); D. G. McDonald, W. Haeberli, and L. W. Morrow, Phys. Rev. 133, B1178 (1964); E. H. Thorndike, Phys. Rev. 138, B586 (1965).

${ }^{7}$ L. N. Blumberg, E. E. Gross, A. van der Woude, A. Zucker, and R. H. Bassel, Phys. Rev. 147, 812 (1966).

${ }^{8}$ L. N. Blumberg, E. E. Gross, A. van der Woude, and A. Zucker, Nucl. Instr. Methods 39, 125 (1966).

\title{
ION-ION POTENTIALS AND THE COMPRESSIBILITY OF NUCLEAR MATTER
}

\author{
Werner Scheid, Rainer Ligensa, and Walter Greiner \\ Department of Physics, University of Virginia, Charlottesville, Virginia, \\ and Institut fur Theoretische Physik der Universität, Frankfurt am Main, Germany
}

(Received 4 September 1968)

With a schematic model for the nuclear matter we give a unified treatment of the real and imaginary parts of the elastic $\mathrm{O}^{16}-\mathrm{O}^{16}$ scattering potential. The model connects the parameters of the potential with the density and binding properties of the $\mathrm{O}^{16}-\mathrm{O}^{16}$ system and reproduces the structure of the excitation function quite well. It is shown that the nuclear compressibility can be obtained from the scattering data, and in the case of the $\mathrm{S}^{32}$ compound system there results an effective compressibility (finite quenching of the nuclei) of about $200 \mathrm{Me} \%$.

In ion-ion collisions, as for example in the elastic $\mathrm{O}^{16}-\mathrm{O}^{16}$ scattering process, the two nuclei penetrate each other if the bombarding energy exceeds the Coulomb barrier (about $12 \mathrm{MeV}$ in the c.m. system for $\left.\mathrm{O}^{16}-\mathrm{O}^{16}\right)$. Above $15 \mathrm{MeV}$ (c.m.) the experimental $90^{\circ} \mathrm{O}^{16}-\mathrm{O}^{16}$ differential cross section found by the Yale group ${ }^{1}$ is depressed by more than a factor 10 from the Mott cross section and shows a regular resonance structure (see Fig. 3). This obviously suggests that a real scattering potential alone cannot describe such a behavior and that a strong imaginary part has to be present. ${ }^{1,2}$ It is the aim of this paper to present some ideas on the origin of the real and imaginary parts of the ion-ion potentials and to compare the results with the experiment.
Before the ions come in contact, the probability for transitions from the $\mathrm{O}^{16}$ ground state remains small since the double-closed $\mathrm{O}^{16}$ shells have no low-lying excitations. Behind the contact point we expect a competition between two processes: the superposition of the densities of the two nuclei and the rearrangement of the $\mathrm{O}^{\mathbf{1 6}}$ shells. Both processes develop in nearly the same time, because the collision time $\tau \approx 5 \times 10^{-22}$ $\mathrm{sec}$ is of the same order of magnitude as a signigicant nuclear time, e.g., the orbital time of a nucleon in the $\mathrm{O}^{16}$ nucleus. In this intermediate region between the limits of an adiabatic and a sudden process we choose the sudden approximation as our starting point. We superpose the nuclear densities to calculate the real part of the potential. The destruction of the $\mathrm{O}^{\mathbf{1 6}}$ shells, by 
which highly excited states of the $\mathrm{S}^{32}$ compound nucleus are formed, will be considered as an essentially irreversible process leading to inelastic scattering. Therefore, we attribute to this latter process the imaginary part of the potential. For its calculation we evaluate the flow of nuclear matter out of regions where the density is compressed and unstable (see Fig. 2).

We propose a simplified and phenomenological model of the nuclear matter similar in the fundamental ideas to those of the recently proposed nuclear matter theories of Bethe ${ }^{3}$ and Brueckner. ${ }^{4}$ The following Ansatz for the total nuclear energy is constructed under the condition that we get a linear and analytically solvable equation for the nuclear density distribution $\rho$ :

$$
E=W_{0} A+\frac{C}{2 \rho_{0}} \int\left(\rho-\rho_{0}\right)^{2} d \tau+\frac{V}{8 \pi} \int \rho\left(\overrightarrow{\mathrm{r}}_{1}\right) \frac{e^{-\left|\overrightarrow{\mathrm{r}}_{1}-\overrightarrow{\mathrm{r}}_{2}\right| / \mu}}{\left|\overrightarrow{\mathrm{r}}_{1}-\overrightarrow{\mathrm{r}}_{2}\right|}\left[\rho\left(\overrightarrow{\mathrm{r}}_{2}\right)-\rho\left(\overrightarrow{\mathrm{r}}_{1}\right)\right] d \tau_{1} d \tau_{2}+\frac{1}{2}\left(\frac{e Z}{A}\right)^{2} \int \frac{\rho\left(\overrightarrow{\mathrm{r}}_{1}\right) \rho\left(\overrightarrow{\mathrm{r}}_{2}\right)}{\left|\overrightarrow{\mathrm{r}}_{1}-\overrightarrow{\mathrm{r}}_{2}\right|} d \tau_{1} d \tau_{2}
$$

The total energy (1) is thus composed of an energy proportional to the particle number $A$, an essentially repulsive energy, an attractive interaction energy $(V<0)$ of Yukawa type with the range $\mu$, and the Coulomb energy in which we put the charge density proportional to the nuclear density.

Disregarding the Coulomb energy and assuming constant density in (1) we obtain the binding energy per nucleon in infinite nuclear matter,

$$
\frac{E}{A}=W_{0}+\frac{1}{2} C \frac{\rho_{0}}{\rho}\left(\frac{\rho}{\rho_{0}}-1\right)^{2} \text {. }
$$

One recognizes the meaning of the constants immediately. $W_{0}$ is the binding energy per nucleon if the density of nuclear matter is in the state of saturation, i.e., if $\rho$ has the equilibrium value $\rho_{0}$. The constant $C$ measures the compressibility of the infinite matter and is connected with the usual compression modulus $K=9 C$.

With the subsidiary condition of constant particle number the variation of the energy with respect to $\rho(\vec{r})$ leads to the following system of linear equations:

$$
\begin{aligned}
& {\left[C / \rho_{0}-V \mu^{2}\right] \rho+\psi+(e Z / A) \varphi=\text { const, }} \\
& \Delta \psi-\psi / \mu^{2}=-V \rho, \\
& \Delta \varphi=-4 \pi(e Z / A) \rho .
\end{aligned}
$$

In the limit of very small densities, $\rho \ll \rho_{0}$, our energy Ansatz (1) is incorrect, as Eq. (2) shows. Therefore, the density cannot steadily fall off to zero in the nuclear surface. From the variation of the energy (1) we also get in addition to the differential equations (3) a boundary condition which cuts the density down at the nuclear surface $(r=R)$ :

$$
\rho(R)=\rho_{0} /\left(1-V \mu^{2} \rho_{0} / C\right)^{1 / 2} .
$$

The general solution for the density distribution has the form

$$
\rho=a j_{0}(\alpha r)+b j_{0}(\beta r) .
$$

Inserting $\rho$ in (3) we find the numbers $\alpha$ and $\beta$, possibly complex, as the roots of a quadratic equation.

With the density distribution of the $\mathrm{O}^{16}$ nucleus obtained in (5) we calculate the real part of the potential for the $\mathrm{O}^{16}-\mathrm{O}^{16}$ elastic scattering. As mentioned before we superpose the densities of the $\mathrm{O}^{16}$ nuclei assuming the sudden approximation:

$$
V(r)=E\left[\rho_{\mathrm{O}^{16}}\left(\overrightarrow{\mathrm{r}}_{1}\right)+\rho_{\mathrm{O}^{16}}\left(\overrightarrow{\mathrm{r}}_{2}\right)\right]-2 B \mathrm{O}^{16},
$$

with the binding energy of $\mathrm{O}^{16},{ }^{B} \mathrm{O}^{16}=E\left(\rho_{\mathrm{O}^{16}}\right)$.

The densities of the $\mathrm{O}^{16}$ nuclei are functions of the distances $\vec{r}_{1}$ and $\vec{r}_{2}$ measured from their centers of mass. All integrals occuring in (6) can be analytically solved for the density (5) by making use of the Fourier transformation of the $\mathrm{Yu}-$ kawa potential.

At large distances only the Coulomb interaction contributes to the potential (Fig. 1). Near the contact point the long-range attractive potential depresses the Coulomb barrier. Finally, if the two nuclei appreciably overlap, a repulsive core arises due to nuclear compressibility. The potential $V(0)$, diminished by the difference of the binding energies of the $\mathrm{S}^{32}$ and the two $\mathrm{O}^{16} \mathrm{nu}$ clei $(16.6 \mathrm{MeV})$, gives the amount of energy needed to compress the mass of the $S^{32}$ nucleus into the shape of the $\mathrm{O}^{16}$ nucleus. To this energy we relate an effective compressibility constant, which includes the influence of the finite size of the nucleus and which turns out to be about two times larger than $K=9 C$, the compressibility of infinite nuclear matter in our schematic model.

In the region of contact of the two ions the su- 


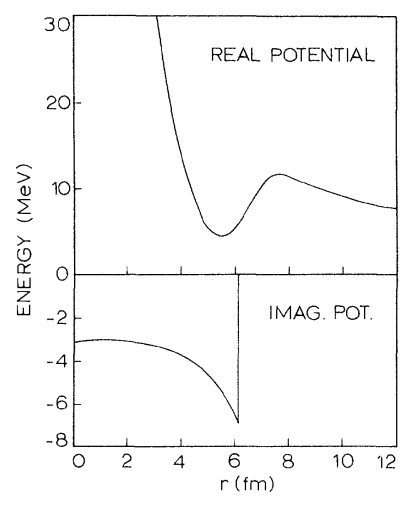

FIG. 1. Real and imaginary part of the potential for $\mathrm{O}^{16}-\mathrm{O}^{16}$ scattering $\left(W_{0}=-14.6 \mathrm{MeV}, \rho_{0}=0.14 / \mathrm{fm}^{3}, C\right.$ $=100 / 9 \mathrm{MeV}, V=-13616 \mathrm{MeV} \mathrm{fm}$, and $\mu=0.3 \mathrm{fm})$. The real potential is $115 \mathrm{MeV}$ for $r=0$.

perposed densities are highly unstable. We expect a flow of nuclear matter out of the regions of compressed densities (see Fig. 2). In a microscopic description we interpret the outflow of matter as the destruction of the $\mathrm{O}^{16}$ shells. The outflow times are comparable with the reaction times of the $S^{32}$ compound system. Therefore, in a never-come-back approximation, the outflow can be thought of as the beginning stage of all inelastic processes.

To connect the imaginary part $W$ of the potential with the characteristic outflow time $T$ we as sume that the time-dependent wave function $\psi$ decreases as

$$
\psi \psi * \sim e^{-t / T} .
$$

Then the imaginary part has the same form as derived with the uncertainty principle of Heisenberg,

$$
W=-i(\hbar / 2 T) .
$$

The outflow time $T$ depends on the degree of overlap of the two $\mathrm{O}^{16}$ nuclei and therefore on their relative distance. To simplify the problem of fixing $T$ we introduce some assumptions:

(a) The nuclear matter flows out in the direction of the smallest resistance, which is the direction perpendicular to the relative motion of the two nuclei (see Fig. 2).

(b) The expansion of the overlap region leads to compression oscillations. The density oscillates about the equilibrium state. Since the outflow of matter is thought of as an irreversible process and our model contains no damping force, we assume an outflow time $T$ of half this oscillator period. This assumption needs further

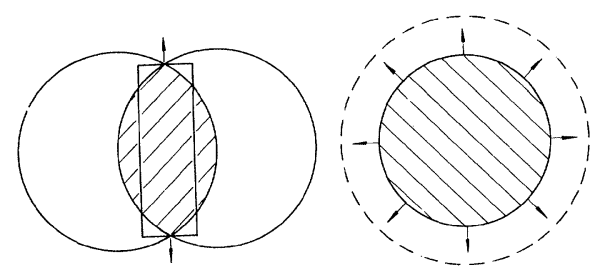

FIG. 2. Model for the imaginary part of the ion-ion potential. The compressed nuclear matter flows out of the region of higher density shown by the shadowed area. The arrows indicate the flow of compressed matter. The cylinder is equivalent to the overlap volume and expands radially to the dotted size drawn in the right-hand figure.

improvement in the future.

(c) To clarify the concept of the oscillator we replace the volume of the overlap region by a cylinder of fixed height as shown in Fig. 2. The radius of the cylinder becomes the oscillator coordinate. Additionally we choose a proper density distribution in the cylinder depending only on the radial coordinate and normalized by the mass in the overlap region.

(d) Instead of expression (1) it is convenient to treat the potential energy of the oscillator by

$$
E_{\text {pot }}=\left(C / 2 \rho_{0}\right) \int\left(\rho-\rho_{0}\right)^{2} d \tau .
$$

In $E_{\text {pot }}$ we neglect the finite range of the attractive potential in (1) and the Coulomb energy.

Near the point of contact the density is too low, no compression takes place yet, and consequently no outflow occurs. Therefore, the imaginary potential starts first at a certain degree of overlap (see Fig. 1). At the starting point of $W$ the described mass cylinder oscillates with a zero amplitude according to the potential energy (9). As the ions overlap more and more, the oscillating mass increases and, consequently, the outflow time $T$ increases which in turn leads to a decrease of the imaginary potential.

Since no further free constants appear in the model of the imaginary part we have only to choose the five parameters $W_{0}, \rho_{0}, C, V$, and $\mu$ of Eq. (1) in order to fix the potentials. Though $W_{0}, \rho_{0}$, and $C$ are approximately known from nuclear matter work, ${ }^{3,4}$ we adjust $W_{0}, \rho_{0}$, and $V$ for a given value of $C$ and $\mu$ to the binding energies of $\mathrm{O}^{16}$ and $\mathrm{S}^{32}(-127.6,-271.8 \mathrm{MeV})$ and to the equivalent radius of $\mathrm{O}^{16}$ or $\mathrm{S}^{32}(3.40,4.10 \mathrm{fm})$. For example with $R_{\mathrm{O}^{16}}=3.4 \overline{\mathrm{fm}}, C=100 / 9 \mathrm{MeV}$, and $\mu=0.8 \mathrm{fm}$ we find $W_{0}=-15.74 \mathrm{MeV}, \rho_{0}=0.154 /$ $\mathrm{fm}^{3}$, and $V=-594 \mathrm{MeV} \mathrm{fm}$. With that the following nuclear properties result in agreement with 


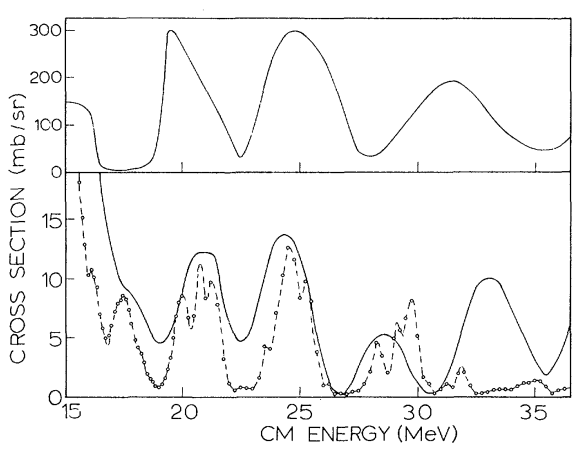

FIG. 3. Comparison of the experimental $90^{\circ}$ (c.m.) differential cross section for elastic $\mathrm{O}^{16}-\mathrm{O}^{16}$ scattering with the theory. The experimental points (open circles) are measured by the Yale group. In the upper half of the figure the theoretical curve is calculated without the imaginary part of the potential, in the lower half with the imaginary part. The parameters of the curves are those of Fig. 1.

experiment: The equivalent radius of $\mathrm{S}^{32}$ is 4.18 $\mathrm{fm}$, the surface thicknesses of $\mathrm{O}^{16}$ and $\mathrm{S}^{32}$ are 1.79 and $2.18 \mathrm{fm}$, respectively, and the specific constant of the surface energy in the mass formula is $17 \mathrm{MeV}$.

The range $\mu$ of the attractive force causes two main effects in the cross section: (a) It influences the absolute value of the cross section sensitively up to $15 \mathrm{MeV}$, since it changes the height of the Coulomb barrier. (b) The position of the maxima and minima above $16 \mathrm{MeV}$ is shifted towards higher energies with decreasing range $\mu$. So we adapt the range $\mu$ by the low-energy elas tic ion-ion cross section and by the position of the resonances.

Finally the last parameter $C$ is used to fix the absolute value of the peaks. With increasing $C$ the imaginary part of the potential also increases, and, therefore, absorption becomes more ef fective. Because the theoretical cross section is sensitive to $C$, this method can be used to determine the nuclear compressibility constant from ion-ion scattering data.

In Fig. 3 we compare our calculations with the experiment. ${ }^{1}$ The potentials used are drawn in Fig. 1. The importance of the imaginary potential is evident. The theoretical cross section is too high in the energy range above $33 \mathrm{MeV}$. Because our calculated imaginary part is energy independent this obviously shows that in the higher energy region energy-dependent inelastic reactions take place. ${ }^{5}$ It is clear that such a crude treatment can only account for the main structure of the excitation function and that the fine structure deserves further investigation.

The constant $C$ is found to be $100 / 9 \mathrm{MeV}$. With that we calculate the effective compressibility constant of the $\mathrm{S}^{32}$ compound system to be 201 $\mathrm{MeV}$. Further details will be published elsewhere. ${ }^{5}$

This work was partly supported by the Deutsche Forschungsgemeinschaft and was carried out under the auspices of the Center for Advanced Studies of the University of Virginia.

${ }^{1}$ R. H. Siemssen, J. V. Maher, A. Weidinger, and D. A. Bromley, Phys. Rev. Letters 19, 369 (1967).

${ }^{2}$ R. J. Munn, B. Block, and F. B. Malik, Phys. Rev. Letters 21, 159 (1968).

${ }^{3}$ H. A. Bethe, Phys. Rev. 167, 879 (1968).

${ }^{4}$ K. A. Brueckner, J. R. Buchler, S. Jorna, and R. J. Lombard, Phys. Rev. 171, 1188 (1968).

${ }^{5}$ W. Scheid, W. Greiner, and R. Ligensa, to be published. 